\title{
Single Balanced Mixer Using Substrate Integrated Waveguide (SIW) $90^{\circ}$ Coupler
}

\author{
Abdelkhalek Nasri, Hassen Zairi, and Ali Gharsallah
}

\begin{abstract}
The present paper focuses on an innovative broadband millimeter- wave single balanced diode mixer which is designed through using a newly designed substrate integrated waveguide (SIW) $90^{\circ}$ coupler. The low cost singlebalanced mixer has its own advantages of low cost, low profile, and high performance. In addition, it has a less conversion loss of $6.6 \mathrm{~dB}$, a wide-band response from 9.5 to $12.5 \mathrm{GHz}$ and better than $-20 \mathrm{~dB}$ port-to-port isolations.
\end{abstract}

Index Terms-Substrate integrated waveguide (SIW), single-balanced mixer, coupler, conversion loss.

\section{INTRODUCTION}

It is well known that the design of modern microwave telecommunication system requires high performance and low-cost mixers. Many passive components such as filters and couplers are incorporated in the design of mixers [1], [2]. In order to minimize the insertion loss and the interference of mixers a good coupler performance is required. However, we cannot forget the low performance of the classical $90^{\circ}$ couplers. That's why, the substrate integrated waveguide (SIW) technology has emerged recently as a substitute for the design of modern microwave and millimeter wave communication systems, thanks to its attractive advantages of low-cost, high quality factor, low insertion loss and high performance [2]-[7].

The electric field distribution in an SIW fills the volume inside the interconnected waveguide and the surface currents propagate on the larger total cross-sectional area of the waveguide walls, thus, it results lower conductor losses. [8]. SIW is considered as a 3-dimensional (3-D) structure for signal transmission, and it seems challenging when it integrates with planar circuits, lines, and connecting pads. In order to address this concern, a host of mode and impedance matching transitions from SIW to microstrip stripline coplanar waveguide (CPW), and conductor backed coplanar waveguide (CBCPW) have been developed recently. We implement all these transitions, like the SIW using the same fabrication process as the rest of system's layout [8], [9].

The millimeter-wave mixers have been subject to intensive studies by researchers particularly in the aspects of port-to-port isolation. It is commonly known that single balanced type mixers have their own advantages of lowprofile, low insertion loss, higher power-handling capabilities and easy integration in SIW circuits [10]-[12]. Moreover, the single balanced mixers made up with a nonlinear devices interrelated by single or multiple hybrids,

Manuscript received July 17, 2014; revised September 16, 2014.

The authors are with the physics Department, Faculty of Sciences of Tunis, El Manar2092, Tunisie (e-mail:abdelkhaleknasri@yahoo.fr, Hassen.zairi@gmail.com, ali.gharsallah@fst.rnu.tn). or transformers.

In This present paper, we will first of all describe the $90^{\circ}$ coupler which is mainly characterized by low conduction loss, then discuss the design of the mixer, and finally present the results and the electrical performances of mixers.

\section{Design of The SIW Coupler}

At the mm-wave the traditional planar hybrid couplers experience an extremely high conduction loss [13]. In addition to their quarter-wave length segments which are shorter than the widths. So obviously, physical implementation and strong mutual coupling difficulties could happen due to this issue.

That's why many passive compact and low cost components have developed rapidly [14]-[17]. For decades, there have been intensive studies of couplers which have been widely used as key components in many systems.

SIW couplers can be used in both microwave and millimeter-wave systems, as well as in modular and in integrated form. However, the present coupler has a simpler design as compared to the narrow wall waveguide coupler.

The configuration of the SIW $90^{\circ}$ coupler is shown in Fig. 1 with its geometry parameters. The spacing ' $a$ ' which stands between the two rows of metallic vias is for the frequency band of the waveguide. The via diameter ' $D$ ' is considered as equal or smaller than a tenth of the wavelength of the maximum operation frequency, while spacing ' $s$ ' is chosen to be equal or smaller than twice the diameter of the metallic via. The geometric parameters are primarily determined by the relationship between the conventional rectangular waveguide and the SIW [1].

A concave area is formed with length ' 12 ' in the middle and depth ' $h$ ' while the length of SIW in each port is ' 13 '. The length between the port and the concave area is ' 11 ', ' $l 1$ ', ' $l 2$ ', ' $w 2$ ', and ' $h$ ' can be changed to control the coupling.

The width ' $t 2$ ' and ' $w 1$ ' and length ' $t$ ' ' of the microstrip taper is adopted to fit the microstrip and SIW in wideband to obtain $50 \Omega$ characteristic impedance [4].

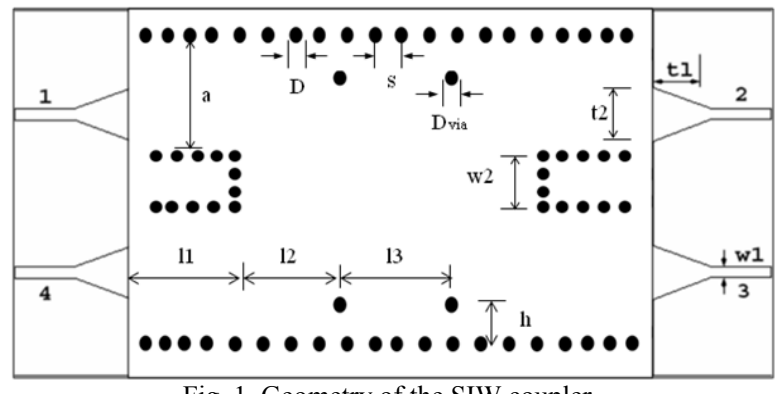

Fig. 1. Geometry of the SIW coupler.

The parameters of the SIW $90^{\circ}$ coupler in Table I are 
obtained after gradual optimization with HFSS (highfrequency structure simulator). It is obvious that the thickness of the dielectric substrate is $0.5 \mathrm{~mm}, \varepsilon_{r}=2.2$ and the dielectric loss tangent is 0.009 .

In order to achieve a wide-band performance, the parameters are finely tuned using three-dimensional (3-D) electromagnetic (EM) simulation software and highfrequency structure simulator (HFSS).

\begin{tabular}{l|l|l|l|l|l}
\hline \multicolumn{7}{|c}{ TABLE I: DIMENSION OF THE STRUCTURE } \\
\hline$a$ & $s$ & $D$ & $t 1$ & $t 2$ & Dvia \\
\hline $12.25 \mathrm{~mm}$ & $0.7 \mathrm{~mm}$ & $0.4 \mathrm{~mm}$ & $20.5 \mathrm{~mm}$ & $7.2 \mathrm{~mm}$ & 0.8 \\
\hline$w 1$ & $w 2$ & $l 1$ & $l 2$ & $l 3$ & $h$ \\
\hline $1.3 \mathrm{~mm}$ & $3.7 \mathrm{~mm}$ & $4.1 \mathrm{~mm}$ & $10.4 \mathrm{~mm}$ & $10.4 \mathrm{~mm}$ & $2.8 \mathrm{~mm}$ \\
\hline
\end{tabular}

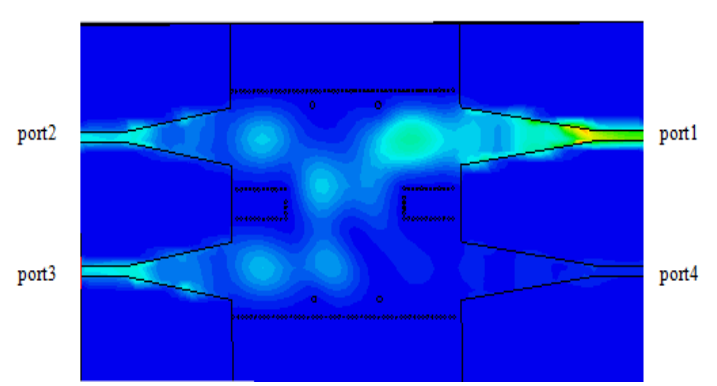

Fig. 2. Electric field distribution of TE10 mode for SIW coupler at $f=12$ $\mathrm{GHz}$.

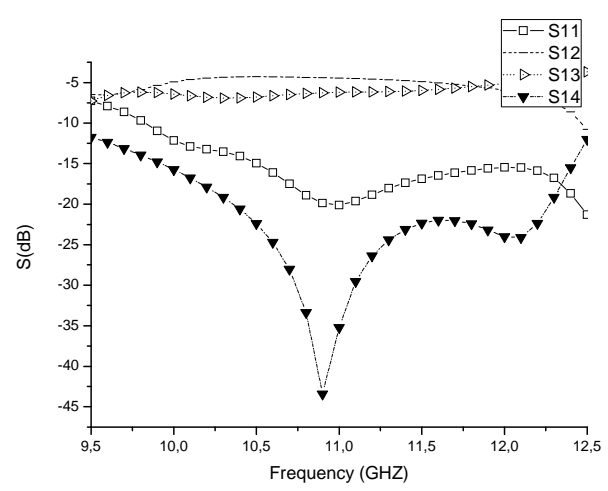

Fig. 3. Simulated S-parameters of SIW coupler.

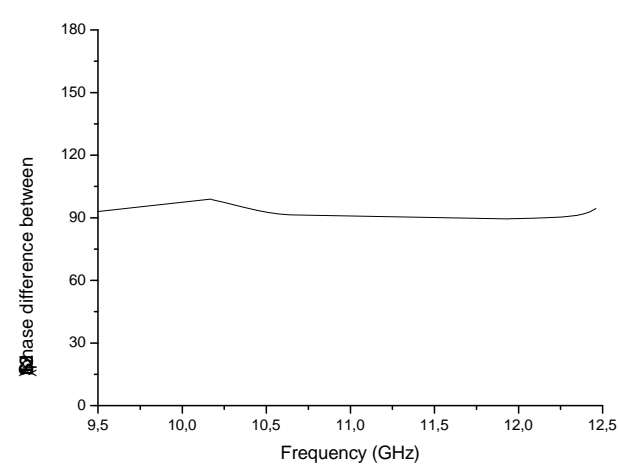

Fig. 4. Simulated phase difference.

The electric field distribution of the TE10 mode, the reflection coefficients $S 11$, the transmission coefficients $S 12$, the coupling coefficient $S 13$ as well as the isolation coefficient $S 14$ are presented in Fig. 2-Fig. 3, respectively. It is noticeable that the $90^{\circ}$ coupler characterizes with good port-to-port isolation. Also, it presents the simulated phase difference between two output ports. It is obvious that $-3 \mathrm{~dB}$ to $-6 \mathrm{~dB}$ are the outputs at ports 2 and 3 . The phase difference is distributed in the range of $89^{\circ}$ to $94^{\circ}$ in the frequency band of 9.5 to $12.5 \mathrm{GHz}$ in Fig. 4 .

So, it is clear that these simulation results demonstrate the good performance of this integrated structure.

\section{Design Of The Single-BALANCEd MiXeR}

In order to get very high frequencies this low-cost and distributed microwave diode mixer, which is very similar to the above mixer, is a good choice when used with Schottky diodes.

Schottky diodes mixer are used in a large number of circuits. The development of these circuits is for enhancing the system performance and optimizing a specific design characteristic. Through combining two Schottky mixer circuits in a balanced configuration where the two diodes are driven in opposite phase, improvement upon the single ended mixer can be obtained.

This extra circuitry in single diode made up of a mixture of passive coupling, power division, and filtering. However, it is hard to make wideband single diode mixer with independent RF, LO, and IF bands while the multiplexing circuitry is frequency specific. In addition, such circuitry engenders extra losses which lead to mixer efficiency reduction [18]-[21].

So, as a solution to these problem researchers found out that wideband single balanced mixers with low loss and independent input and output frequency bands could be created using the SIW $90^{\circ}$ coupler. This latter includes two inputs, two output, and four port circuit that provides mutual isolation between input ports and equal power division at the output ports [22], [23]. It makes clear that the hybrid junction for mixers is applicable as follows: the input LO and RF sources will be isolated from one another, which provide frequency band independence and equal power division to the load.

Fig. 5 presents a single balanced mixer with two diodes connected back-to-back, in addition to an SIW coupler formed on the same dielectric substrate. The SIW coupler performs as the input and power delivery component better than the classical couplers. Thanks to SIW structure, the insertion loss and interference on other circuits is minimized due to the radiation loss which exists only at the transitions between the SIW coupler and microstrip. In addition, the performance of the mixer is enhanced by designing carefully the coupler and transitions, in which the $90^{\circ} \mathrm{SIW}$ coupler shows a considerably wide band performance at a desired frequency.

The production of the difference frequency when two frequencies are combined or mixed in the diode is considered as the primarily application of the Schottky diode. Besides, this combination or mixing action is due to the non-linear relationship between current and voltage.

The mixing output signal at the output port made up with a signal whose designed frequency is the difference between RF frequency and LO frequency and this desired signal named intermediate frequency (IF) along with other 
unwanted signal products.

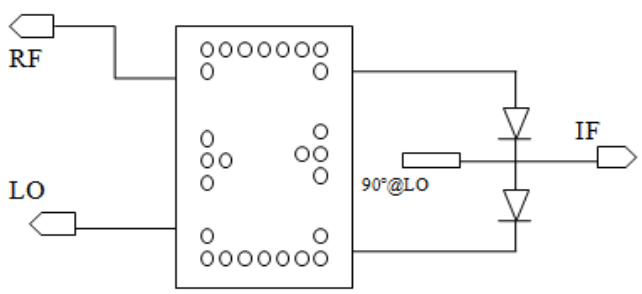

Fig. 5. Configuration of the proposed SIW mixer.

\section{Simulated Results}

This part focuses on the simulation results of the single balanced mixer. Analysis results of SIW coupler from HFSS are included in the harmonic balance simulation through using "S4P" data item of ADS (Advanced Design System).

The major design issue in passive mixer is to reduce the conversion loss. Through the simulation results we can prove the good performance of this integrated structure. Fig. 6 and Fig. 7 shows the simulated results. Where the RF frequency is $12 \mathrm{GHz}$, the LO frequency is $10 \mathrm{GHz}$, the LO power sweep from -10 to $10 \mathrm{dBm}$, the RF power is $-30 \mathrm{dBm}$, and the simulated conversion losses are under $6.6 \mathrm{~dB}$ with LO power $8 \mathrm{dBm}$. The input power causes a drop of $1 \mathrm{~dB}$ in the linear gain due to device saturation. Fig. 7 shows the $1 \mathrm{~dB}$ compression point which is $12 \mathrm{~dB}$ of $\mathrm{RF}$ input power.

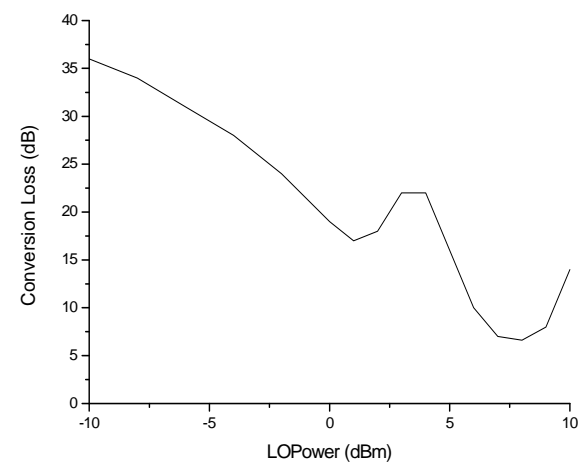

Fig. 6. Conversion loss versus LO power.

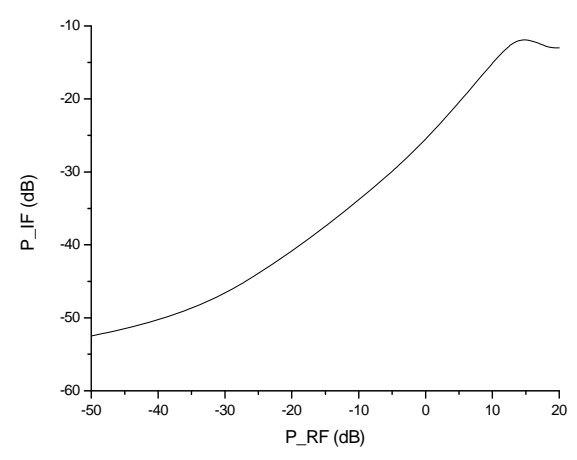

Fig. 7. P1_dB gain compression.

\section{CONCLUSION}

In this paper, we suggested a low cost single balanced microwave mixer design using a $90^{\circ}$ SIW Coupler. The simulated results show a low conversion loss and a high performance. Our proposed mixer can guarantee a good port-to-port isolation.

So, it is noticeable that the suggested mixer has a good advantages that help design a high performance microwave and millimeter wave integrated circuits. Noting that, an antenna can be integrated directly with our design in order to structure a very compact receiver front-end of millimeterwave communication system.

\section{REFERENCES}

[1] Y. L. Zhang, W. Hong, F. Xu, K. Wu, and T. J. Cui, "Analysis of guided wave problems in substrate integrated waveguides - numerical simulation and experimental results," IEEE MTT-S Int. Dig., vol. 3, Jun. 2003, pp. 2049-2052.

[2] H. Li, W. Hong, T. J. Cui, K. Wu, Y. L. Zhang, and L. Yan, "Propagation characteristics of substrate integrated waveguide," IEEE MTT-S Int. Dig., vol. 3, Jun. 2003, pp. 2045-2048.

[3] K. Wu, D. Deslandes, and Y. Cassivi, "The substrate integrated circuits a new concept for high-frequency electronics and optoeletronics," in Proc. the 6th Int. Conf. Telecommunications Modern Satellite, Cable Broadcasting Service (TELSIKS'03), vol. 1, Oct. 1-3,2003, pp. P-III-P-X.

[4] J. X. Chen, W. Hong, Z. C. Hao, H. Li, and K. Wu, "Development of a low cost microwave mixer using a broad-band substrate integrated waveguide (SIW) coupler," IEEE Microwave And Wireless Components Letters, vol. 16, no. 2, 2006.

[5] Z. Y. Zhang, Y. R. Wei, and K. Wu, "Broadband millimeter-wave single balanced mixer and its applications to substrate integrated wireless systems," IEEE Transactions on Microwave Theory and Techniques, vol. 60, no. 3, 2012.

[6] A. Suntives and R. Abhari, "Experimental evaluation of a hybrid substrate integrated waveguide," IEEE, pp. 1-9, 2008.

[7] W. M. A. Wahab and S. S. Naeini, "Low loss h-shape SIW hybrid coupler for millimeter-wave phased arrays antenna systems," IEEE, pp. 1-2, 2012.

[8] A. Patrovsky, M. Daigle, and K. Wu, "Millimeter-wave wideband transition from CPW to substrate integrated waveguide on electrically thick high-permittivity substrates," in Proc. the Microwave Conference, pp. 138-141, 2007.

[9] D. Deslandes and $\mathrm{K}$. Wu, "Integrated microstrip and rectangular waveguide in planar form," IEEE Microw. Wireless Compon. Lett., vol. 11 , no. 2 , pp. $68-70$, Feb. 2001.

[10] J. J. Xu and K. Wu," A subharmonic self-oscillating mixer using substrate integrated waveguide cavity for millimeter-wave application," in Proc. IEEE Conference on Microwave Symposium Digest, 2005, pp. 12-17.

[11] Y. T. Wang, X. W. Zhu, C. J. You, and G. Q. Yang, "Design of kaband half mode substrate integrated waveguide (HMSIW) mixer," in Proc. IEEE Conference on Ultra-Wideband, 2009, pp. $684-687$.

[12] L. Han, K. Wu, and S. Winkler, "Singly balanced mixer using substrate integrated waveguide magic-t structure," in Proc. the 1st European Wireless Technology Conference October, pp. 9-12, 2008.

[13] P. Meaney, "A novel branch-line coupler design for millimeter-wave applications," IEEE Microwave International Symposium Digest, MTTS, vol. 1, pp. 585-588, 1990.

[14] N. A. Smith, "Substrate integrated waveguide circuits and systems," Thesis for the degree of Master of Engineering, Department of Electrical and Computer Engineering McGill University Montréal, Québec, Canada , May 2010.

[15] T. Coenen, "Analysis and design of post wall waveguides for applications in SIW," PhD Thesis, the Telecommunication Engineering group of the Faculty of Electrical Engineering, Mathematics and Computer Science of the University of Twente Enschede, The Netherlands, 2010.

[16] J. J. Xu and K. Wu, "A subharmonic self-oscillating mixer using substrate integrated waveguide cavity for millimeter-wave application," IEEE Conference on Microwave Symposium Digest, p. $10,2005$.

[17] T. Djerafi and K. Wu, "Super-compact substrate integrated waveguide cruciform directional coupler," IEEE Microwave and Wireless Components Letters, vol. 17, no. 11, 2007.

[18] Z. Y. Zhang, K. Wu, and N. Yang," A millimeter-wave sub-harmonic self-oscillating mixer using dual-mode substrate integrated waveguide cavity," IEEE Transactions on Microwave Theory and Techniques, vol. 58, no. 5, 2010.

[19] Y. W. Wang, J. Y. Zhou, and W. Hong, "Study of dual-balanced mixer with half mode substrate integrated waveguide," in Proc. the 
International Conference on Material and Manufacturing Technology, vol. 3, 2008 .

[20] J. X. Chen, W. Hong, H. J. Tang, X. X. Yin, and K. Wu, “A compact millimeter-wave mixer module," in Proc. International Conference on $A P M C$, vol. 5, 2005.

[21] S. Raman, F. Rucky, and G. M. Rebeiz, "A high-performance w-band uniplanar subharmonic mixer," IEEE Trans. Microwave Theory Tech., vol. 45, pp. 955-962, June.1997.

[22] Z. Y. Zhang, K. Wu, and Y. R. Wei, "180-degree substrate integrated waveguide hybrid and its application to broadband millimeter-wave single balanced mixer design," in Proc. the Asia-Pacific Microwave Conference, pp. 1649-1652, 2010.

[23] Q. Xue, K. M. Shum, and C. H. Chan, "Low conversion-loss fourth subharmonic mixers incorporating CMRC for millimeter-wave applications," IEEE Transactions on Microwave Theory and Techniques, vol. 51, no. 5, 2003.

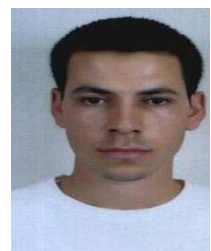

Abdelkhalek Nasri received his master's degree in electronics from the Faculty of Sciences, Tunis, Tunisia, in 2011. He is currently working toward his $\mathrm{PhD}$ degree at the Faculty of Sciences, Tunis. His research interests include microwave integrated circuits.

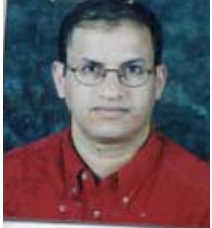

Hassen Zairi received his master's degree in physics from the Faculty of Sciences, Tunis, Tunisia, in 2002 and the $\mathrm{PhD}$ degree in 2005 from the Faculty of Sciences, Tunis. His research interests include antenna for wireless communication and microwave integrated circuits.

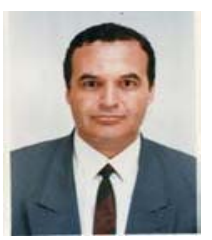

Ali Gharsallah is currently a full professor of electrical engineering and Director of Engineering in the Higher Ministry Education of Tunisia. He is the author of about 55 papers published in scientific journals and 80 conference papers. His current research interests include antennas, multilayered structures and microwave integrated circuits. 